\title{
Association Between Bullying and Suicidal Behavior Among Chinese Adolescents: An Analysis of Gender Differences
}

This article was published in the following Dove Press journal: Psychology Research and Behavior Management

\author{
Tingting Yang' \\ Lan Guo ${ }^{2}$ \\ Feng Hong' \\ Ziyun Wang' \\ Yao $\mathrm{Yu}^{3}$ \\ Ciyong $\mathrm{Lu}^{2}$
}

'School of Public Health, The Key Laboratory of Environmental Pollution Monitoring and Disease Control, Ministry of Education, Guizhou Medical University, Guiyang 550025, People's Republic of China; ${ }^{2}$ Department of Medical Statistics and Epidemiology, School of Public Health, Sun Yat-Sen University, Guangzhou 510080, People's Republic of China; ${ }^{3}$ The Affiliated Hospital of Guizhou Medical University, Guiyang 550004, People's Republic of China

Correspondence: Ciyong Lu Department of Medical Statistics and Epidemiology, School of Public Health, Sun Yat-Sen University, Guangzhou 510080 , People's Republic of China Tel +86-20-87332477

Email luciyong@mail.sysu.edu.cn
Background: Suicide has become the leading cause of death among 15-34 years old in China, and more attention should be paid to risk factors and prevention of suicidal ideation and suicide attempts. The school-based survey was conducted to test the associations of bullying behavior with suicidal ideation and suicide attempts, and to assess whether the biological gender can moderate these associations.

Methods: A multistage stratified cluster sampling method was used to recruit participants from high schools in Guizhou, China, and univariate and multivariate logistic regression models were fitted.

Results: A total of 23,392 students were included in the analysis, and $45.4 \%$ were boys. The mean age of the students was 15.2 (SD: 1.9) years. Overall, 3.8\% (882) of the students were victims, $4.9 \%$ (1144) were bullies, and 3.2\% (736) were bully-victims. A total of $18.9 \%$ of students reported having suicidal ideation, and 3.9\% reported having suicide attempts. Victims (adjusted odds ratio $[\mathrm{AOR}]=2.15,95 \% \mathrm{CI}=1.84-2.51$ ), bullies (AOR $=3.17,95 \%$ $\mathrm{CI}=2.78-3.62)$, and bully-victims $(\mathrm{AOR}=3.18,95 \% \mathrm{CI}=2.71-3.73)$ were at an elevated risk of suicidal ideation than neutrals; similar associations can be found in the associations between bullying behavior and suicide attempts. Further stratification analyses showed that the associations of being bullied or bullying others with suicidal ideation and suicide attempts were slightly stronger in girls than in boys.

Conclusion: Prevention or intervention programs are recommended to take the biological gender into consideration, and future studies are also warranted to investigate the reasons of differences by gender in the association of bullying and suicidal behavior.

Keywords: bullying behavior, suicidal ideation, Chinese adolescents, gender difference

\section{Introduction}

Suicidal behavior, ranging from suicidal ideation (having thoughts about killing oneself), suicide attempts (engagement in potentially self-injurious behavior in which there is some intent to die) to suicide death, is a leading cause of injury and death worldwide. ${ }^{1,2}$ Although the overall suicide death rate in China has been decreased from the ratio in the $1990 \mathrm{~s},{ }^{3}$ suicidal ideation and suicide attempts are still a significant public health concern among Chinese adolescents, and a prior study demonstrated that approximately $16.1 \%$ of the Chinese adolescents reported having suicidal ideation, and $3.1 \%$ reported having suicide attempts during the last year. ${ }^{4}$ Suicidal ideation and suicide attempts are more common than suicide death and are considered risk factors for suicide death among adolescents. ${ }^{5}$ Moreover, suicidal ideation and suicide attempts 
among adolescents can lead to a substantial financial, social, and psychological burden for the individuals, families, and communities; ${ }^{6}$ girls were reported to have higher rates of suicidal behavior than boys. ${ }^{7}$ The causes of suicidal behavior are multifactorial, including many factors associated with psychological, biological, school, or social domains., 8

Bullying (i.e., bullying others, being bullied, and both bullying others and being bullied) includes teasing, verbal or written name-calling, threats, and social exclusion, as well as kicking, hitting, or another violent bodily contact, which is a common issue among adolescents worldwide. ${ }^{10}$ Recent attention has focused on the association between adolescent bullying and suicidal behavior, reflected in both the public health and research areas. ${ }^{11}$ Previous evidence reported that adolescents who were involved in bullying in any capacity, including victims, perpetrators, and those who are both victims and perpetrators (i.e., bully-victims), were at a higher risk of suicidal ideation and suicide attempts. ${ }^{12,13}$ Additionally, some studies reported that both bullying others and being bullied have emerged as the most influential factor for suicidal behaviors, ${ }^{14,15}$ but this association has not been confirmed by other studies. $^{13,16}$

Evidence suggested that boys were more likely to be bullies, and girls were more involved as victims. ${ }^{17}$ Moreover, several studies have demonstrated that the association between bullying and suicidal behavior may vary by the biological gender. ${ }^{18,19}$ Klomek et al reported higher suicide risk for girls who are victims of bullying, ${ }^{18}$ Laukkanen et al found a stronger association between victimization and suicidal ideation, ${ }^{19}$ and $\mathrm{Kim}$ et al reported non-significant associations between bullying and suicidal ideation for both boys and girls. ${ }^{20}$ Therefore, the biological gender may play a moderator in the association between bullying and suicidal behavior among adolescents. Due to the differences of social and cultural background between China and western or developed countries, we conducted this large-scale study aimed to test the associations between three types of bullying (bullying victimization, bullying perpetration, and bully/victim status) and suicidal behavior (suicidal ideation and suicide attempts), and to assess whether the biological gender can moderate these associations.

\section{Methods}

\section{Study Design and Participants}

A cross-sectional study was conducted in 2014 in Guizhou province, located in southwest China. A multistage stratified cluster sampling method was adopted to obtain a representative sample. In stage 1, Guizhou province was divided into three economic stratifications (high-economic, middle-economic, and low-economic levels) according to per capita gross domestic product in 2013. Then, we randomly selected two representative districts (or primary sampling units) from each stratification. In stage 2, a total of four general and four vocational high schools were randomly chosen from each selected district. In stage 3 , two classes (or minimum sampling units) were randomly selected from each grade within the selected schools. All available students in the selected classes were invited to participate in this study voluntarily. Of the total students who were invited, 23,391 student's questionnaires were completed and qualified for the survey, resulting in a response rate of $95.4 \%$. A rigorously anonymous method for collecting self-report data was guaranteed to protect the student's privacy. The study received approval from the Sun Yat-sen University, School of Public Health Institutional Review Board. Written informed consent letters were obtained from each participating student who was at least 18 years of age. If the student was under 18 years of age, the purpose and nature of the study were explained to the student and his/her parents (or legal guardians), and a written informed consent letter was obtained from one of the student's parents (or legal guardians). This study was conducted in accordance with the Declaration of Helsinki.

\section{Measures}

\section{Suicidal Ideation and Suicide Attempts}

Suicidal ideation was assessed by the question: "How many times did you consider attempting suicide during the past 12 months?" Responses were coded as zero and once or more. Suicide attempt was defined as responding "one or more times" to the question: "How many times did you actually attempt suicide during the past 12 months?",21,22

\section{Bullying Behaviors}

Bullying behaviors were assessed using the 12 questions adapted from a previous study, ${ }^{16}$ which has been widely used in prior studies among Chinese adolescents. ${ }^{23}$ In the present study, the Cronbach's alpha of the bullying items was 0.80 , and that of victimization was 0.74 . These questions were about how frequently the students had been bullied/ bullied others during the last month, with the answers given on a 3-point scale as follows: one $=$ never, two $=$ sometimes or rarely (one or more times), and three $=$ often (more than three times). The six bullying items were posed: "Have you ever threatened another student or say something mean to him or 
her?"; "Have you ever hit, pushed, or hurt another student?"; "Have you ever made fun of or teased another student in a hurtful way?"; "Have you ever made fun of another student with sexual jokes, comments, or gestures?"; "Have you ever blackmailed another student for money?"; "Have you ever bullied someone in other ways?". The six being bullied questions were the following: "Have you ever been threatened or said something mean by another student?"; "Have you ever been hit, pushed, or hurt by another student?"; "Have you ever been made fun of or teased in a hurtful way by another student?"; "Have you ever been made fun of with sexual jokes, comments, or gestures by another student?"; "Have you ever been blackmailed for money by another student?"; "Have you ever been bullied by another student in some other way?". Bullies were the students who reported at least one type of bullying behavior with a frequency of "often". Students who reported at least one being bullied experience with a frequency of "often" were classified as victims. Bully-victims were those who met the criteria for bullying others and also being bullied, with a frequency of "often", and neutrals were students who reported having never been bullied nor bullying others.

\section{Other Characteristics}

Information about gender, age, living arrangement, household socioeconomic status (HSS), classmate relations, teacherclassmate relations, academic performance, smoking, and drinking were also collected. Living arrangement was measured by asking students whom they lived with all or most of the time. Household socioeconomic status (HSS) was assessed by asking students to rate their perceptions of their family economic status. Classmate relations and teacher-classmate relations were measured based on the student's perception. Considering all the recruited students know their place in academic performance (each grade in each recruited high school has the recorded ranking of the final exam at the academic semester, and this record is always distributed to students), academic performance was measured by asking the students to report their ranking in the final exam at the academic semester closest to the survey period. ${ }^{24}$ Smoking or drinking was evaluated by asking students the question: "have you smoked a cigarette (or drunk alcohol) in your lifetime?"

\section{Statistical Analysis}

First, descriptive analyses stratified by gender were used to describe sample characteristics. The chi-square test for categorical variables and the $T$-test for the continuous variables were used to compare differences between boys and girls. Second, all demographic variables were simultaneously entered into the multivariate logistic regression models to estimate the adjusted association of bullying behavior with suicidal ideation and suicide attempts. Third, multiplicative interaction items were tested by entering a cross-product term for bullying behavior and gender along with the main effect terms for each to the multivariate logistic regression models, and $P$-values for the multiplicative interaction were calculated. If the interaction item was significantly associated with suicidal ideation and suicide attempts, stratification analyses would be conducted to assess whether the associations of bullying behavior with suicidal ideation and suicide attempts varied with the student's gender. The percentage of missing data was less than $0.6 \%$ for all relevant variables. Regarding the logistic regression models, observation with missing data was eliminated. All statistical tests were twosided, and $P$-values less than 0.05 were considered statistically significant. All statistical analyses were conducted using Stata SE 12.0 (StataCorp, Houston, Texas, USA).

\section{Results}

\section{Demographic Characteristics of the Study Sample}

As shown in Table 1, of the total sample, 45.4\% (10,625) were boys, and $54.6 \%(12,767)$ were girls. The mean age of the students was 15.2 (SD: 1.9) years, and the age range of the students was 12 to 19 years. The majority of students lived in two-parent families $(66.0 \%)$, and the proportion of students who reported above-average HSS was $10.7 \%$. The proportion of students who reported above-average academic performance, poor classmate relations, and poor relationships with teachers was $28.6 \%, 2.1 \%, 5.0 \%$, respectively. Approximately $10.7 \%$ of students admitted smoking, and $52.2 \%$ reported drinking. Overall, 3.8\% (882) of the students were victims, $4.9 \%$ (1144) were bullies, and 3.2\% (736) were bully-victims. A total of $18.9 \%$ of students reported having suicidal ideation, and $3.9 \%$ reported having suicide attempts. There were significant differences between boys and girls in the distribution of living arrangement, age, HSS, academic performance, classmate relations, relationships with teachers, smoking, drinking, bullying behavior, suicidal ideation, and suicide attempts $(P<0.05)$.

\section{Association of Bullying Behavior and Gender with Suicidal Ideation and Suicide Attempts}

Without adjusting for other variables, bullying behavior and gender were significantly associated with suicidal ideation 
Table I Sample Characteristics Stratified by the Students' Gender (N=23,392)

\begin{tabular}{|c|c|c|c|c|}
\hline Variable & Total, n (\%) & Boys, n (\%) & Girls, n (\%) & $P$-value $\#$ \\
\hline \multicolumn{5}{|l|}{ Living arrangement } \\
\hline Living in two-parent family & $|5,38|(66.0)$ & $6839(64.7)$ & $8542(67.2)$ & $<0.001$ \\
\hline Living in a single-parent family & $3102(13.3)$ & $1446(13.7)$ & $1656(13.0)$ & \\
\hline Living with others & $4804(20.6)$ & $2284(21.6)$ & $2520(19.8)$ & \\
\hline Missing data & 105 & & & \\
\hline Age, mean (SD) & $15.2(1.9)$ & $15.2(1.9)$ & I5.3 (I.8) & $<0.001$ \\
\hline \multicolumn{5}{|l|}{ HSS } \\
\hline Above average & $2484(10.7)$ & $1300(12.3)$ & $1184(9.3)$ & $<0.001$ \\
\hline Average & 13,453 (57.7) & $6050(57.2)$ & $7403(58.2)$ & \\
\hline Below average & $7369(31.6)$ & $3232(30.5)$ & $4137(32.5)$ & \\
\hline Missing data & 86 & & & \\
\hline \multicolumn{5}{|l|}{ Academic performance } \\
\hline Above average & $667 \mid(28.6)$ & $3007(28.4)$ & $3664(28.8)$ & $<0.001$ \\
\hline Average & $8967(38.4)$ & 3470 (32.7) & $5497(43.2)$ & \\
\hline Below average & $7685(33.0)$ & $4120(38.9)$ & $3565(28.0)$ & \\
\hline Missing data & 69 & & & \\
\hline \multicolumn{5}{|l|}{ Classmate relations } \\
\hline Poor or fair & $499(2.1)$ & $289(2.7)$ & $210(1.6)$ & \\
\hline Average & $8945(38.3)$ & $3621(34.2)$ & $5324(41.8)$ & \\
\hline Excellent or good & I3,890 (59.5) & $6688(63.1)$ & $7202(56.5)$ & \\
\hline Missing data & 58 & & & \\
\hline \multicolumn{5}{|l|}{ Relationships with teachers } \\
\hline Poor or fair & $1159(5.0)$ & $762(7.2)$ & $397(3.1)$ & $<0.001$ \\
\hline Average & I3,628 (58.4) & $5695(53.8)$ & $7933(62.3)$ & \\
\hline Excellent or good & $8533(36.6)$ & $4134(39.0)$ & $4399(34.6)$ & \\
\hline Missing data & 72 & & & \\
\hline \multicolumn{5}{|l|}{ Smoking } \\
\hline No & $20,885(89.3)$ & $8568(80.6)$ & $12,317(96.5)$ & $<0.001$ \\
\hline Yes & $2507(10.7)$ & $2057(19.4)$ & $450(3.5)$ & \\
\hline \multicolumn{5}{|l|}{ Drinking } \\
\hline No & II,I93 (47.8) & $4073(38.3)$ & $7 \mid 20(55.8)$ & $<0.001$ \\
\hline Yes & $12,199(52.2)$ & $6552(61.7)$ & $5647(44.2)$ & \\
\hline \multicolumn{5}{|l|}{ Bullying behavior } \\
\hline Neutral & $20,48 I(88.1)$ & 8711 (82.7) & $11,770(92.6)$ & $<0.001$ \\
\hline Victim & $882(3.8)$ & $607(5.8)$ & $275(2.2)$ & \\
\hline Bully & II 44 (4.9) & $700(6.6)$ & $444(3.5)$ & \\
\hline Bully-victim* & $736(3.2)$ & $515(4.9)$ & $221(1.7)$ & \\
\hline Missing data & 149 & & & \\
\hline \multicolumn{5}{|l|}{ Suicidal ideation } \\
\hline No & $18,963(81.1)$ & $8899(83.8)$ & $10,064(78.8)$ & $<0.001$ \\
\hline Yes & $4429(18.9)$ & $1726(16.2)$ & $2703(21.2)$ & \\
\hline \multicolumn{5}{|l|}{ Suicide attempts } \\
\hline No & $22,491(96.1)$ & $|0,26|(96.6)$ & $12,230(95.8)$ & 0.002 \\
\hline Yes & 901 (3.9) & $364(3.4)$ & $537(4.2)$ & \\
\hline
\end{tabular}

Notes: *Bully-victim represents both bullying others and being bullied. ${ }^{\#}$ The chi-square tests were used for a categorical variable, and T-tests were used for continuous data. Abbreviations: SD, standard deviation; HSS, household socioeconomic status. 
and suicide attempts among students. After adjusting for gender, living arrangement, age, HSS, academic performance, classmate relations, relationships with teachers, smoking, and drinking, victims (adjusted odds ratio $[\mathrm{AOR}]=2.15,95 \%$ $\mathrm{CI}=1.84-2.51)$, bullies ( $\mathrm{AOR}=3.17,95 \% \quad \mathrm{CI}=2.78-3.62)$, and bully-victims $(\mathrm{AOR}=3.18,95 \% \mathrm{CI}=2.71-3.73)$ were at an elevated risk of suicidal ideation than neutrals; similar associations can be found in the associations between bullying behavior and suicide attempts (Table 2).

\section{Association of Bullying Behavior with Suicidal Ideation and Suicide Attempts: Moderated by the Students' Gender}

First, the interaction item (between bullying behavior and gender) was significantly associated with suicidal ideation and suicide attempts $(P$ for suicidal ideation $=0.019$ and $P$ for suicide attempts $=0.015$ ). Then, further stratification analyses by the students' gender were performed. In Table 3, multivariate logistic regression models demonstrated that among boys, being bullied, bullying others, and both bullying others and being bullied were positively associated with suicidal ideation and suicide attempts; similar associations can be found in the associations of bullying behavior with suicidal ideation and suicide attempts among girls. The associations of being bullied with suicidal ideation and suicide attempts were slightly stronger in girls (suicidal ideation: $\mathrm{AOR}=2.25$, 95\% $\mathrm{CI}=1.74-2.91$; suicide attempts: $\mathrm{AOR}=2.60,95 \%$ $\mathrm{CI}=1.76-3.84$ ) than in boys (suicidal ideation: $\mathrm{AOR}=2.08$, 95\% $\mathrm{CI}=1.71-2.53$; suicide attempts: $\mathrm{AOR}=2.22,95 \%$ $\mathrm{CI}=1.56-3.18$ ), and the associations of bullying others with suicidal ideation and suicide attempts were also slightly greater in girls (suicidal ideation: $\mathrm{AOR}=3.34,95 \% \mathrm{CI}=2.73-4.08$; suicide attempts: $\mathrm{AOR}=3.74,95 \% \mathrm{CI}=2.76-5.07)$ than in boys (suicidal ideation: $\mathrm{AOR}=2.95,95 \% \mathrm{CI}=2.47-3.53$; suicide attempts: $\mathrm{AOR}=2.92,95 \% \mathrm{CI}=2.10-4.05)$. Moreover, the associations of both bullying others and being bullied with

Table 2 Association of Bullying Behavior and Gender with Suicidal Ideation and Suicide Attempts

\begin{tabular}{|c|c|c|c|c|c|c|c|c|}
\hline \multirow[t]{3}{*}{ Variable } & \multicolumn{4}{|l|}{ Model I } & \multicolumn{4}{|l|}{ Model 2} \\
\hline & \multicolumn{2}{|c|}{ Suicidal Ideation } & \multicolumn{2}{|c|}{ Suicide Attempts } & \multicolumn{2}{|c|}{ Suicidal Ideation } & \multicolumn{2}{|c|}{ Suicide Attempts } \\
\hline & OR (95\% Cl) & $P$-value & OR $(95 \% \mathrm{CI})$ & $P$-value & AOR $(95 \% \mathrm{Cl})$ & $P$-value & AOR (95\% Cl) & $P$-value \\
\hline Bullying behavior & & & & & & & & \\
\hline Neutral & I.00 (reference) & & 1.00 (reference) & & 1.00 (reference) & & 1.00 (reference) & \\
\hline Victim & $2.40(2.07-2.78)$ & $<0.001$ & $3.19(2.50-4.08)$ & $<0.001$ & $2.15(I .84-2.5 I)$ & $<0.001$ & $2.37(1.83-3.08)$ & $<0.001$ \\
\hline Bully & $3.10(2.74-3.5 \mathrm{I})$ & $<0.001$ & $3.50(2.83-4.33)$ & $<0.001$ & $3.17(2.78-3.62)$ & $<0.001$ & $3.41(2.73-4.26)$ & $<0.001$ \\
\hline Bully-victim* & $3.46(2.97-4.02)$ & $<0.001$ & $5.36(4.28-6.70)$ & $<0.001$ & $3.18(2.7 I-3.73)$ & $<0.001$ & $4.30(3.39-5.45)$ & $<0.001$ \\
\hline \multicolumn{9}{|l|}{ Gender } \\
\hline Boys & I.00 (reference) & & 1.00 (reference) & & NA & & NA & \\
\hline Girls & $1.39(1.30-1.48)$ & $<0.001$ & $1.24(1.08-1.42)$ & 0.002 & NA & & NA & \\
\hline
\end{tabular}

Notes: Model I was unadjusted models. Model 2 was adjusted for gender, living arrangement, age, household socioeconomic status, academic performance, classmate relations, relationships with teachers, smoking, and drinking. *Bully-victim represents both bullying others and being bullied.

Abbreviations: OR, odds ratio; $95 \% \mathrm{Cl}, 95 \%$ confidence interval; AOR, adjusted odds ratio; NA, not applicable or no data available.

Table 3 Association of Bullying Behavior with Suicidal Ideation and Suicide Attempts: Moderated by the Students' Gender

\begin{tabular}{|c|c|c|c|c|c|c|c|c|}
\hline \multirow[t]{3}{*}{ Variable } & \multicolumn{4}{|c|}{ Suicidal Ideation ${ }^{\#}$} & \multicolumn{4}{|c|}{ Suicide Attempts } \\
\hline & \multicolumn{2}{|l|}{ Boys } & \multicolumn{2}{|l|}{ Girls } & \multicolumn{2}{|l|}{ Boys } & \multicolumn{2}{|l|}{ Girls } \\
\hline & AOR $(95 \% \mathrm{Cl})$ & $P$-value & AOR (95\% Cl) & $P$-value & AOR $(95 \% \mathrm{Cl})$ & $P$-value & AOR $(95 \% \mathrm{Cl})$ & $P$-value \\
\hline Bullying behavior & & & & & & & & \\
\hline Neutral & I.00 (reference) & & I.00 (reference) & & 1.00 (reference) & & I.00 (reference) & \\
\hline Victim & $2.08(1.71-2.53)$ & $<0.001$ & $2.25(1.74-2.91)$ & $<0.001$ & $2.22(1.56-3.18)$ & $<0.001$ & $2.60(1.76-3.84)$ & $<0.001$ \\
\hline Bully & $2.95(2.47-3.53)$ & $<0.001$ & $3.34(2.73-4.08)$ & $<0.001$ & $2.92(2.10-4.05)$ & $<0.001$ & $3.74(2.76-5.07)$ & $<0.001$ \\
\hline Bully-victim* & $3.16(2.59-3.85)$ & $<0.001$ & $3.16(2.39-4.17)$ & $<0.001$ & $4.47(3.28-6.08)$ & $<0.001$ & $3.82(2.62-5.58)$ & $<0.001$ \\
\hline
\end{tabular}

Notes: \#Models were adjusted for living arrangement, age, household socioeconomic status, academic performance, classmate relations, relationships with teachers, smoking, and drinking. *Bully-victim represents both bullying others and being bullied.

Abbreviations: AOR, adjusted odds ratio; $95 \% \mathrm{Cl}, 95 \%$ confidence interval. 
suicidal ideation were similar between boys (suicidal ideation: $\mathrm{AOR}=3.16,95 \% \mathrm{CI}=2.59-3.85$ ) and girls (suicidal ideation: $\mathrm{AOR}=3.16,95 \% \mathrm{CI}=2.39-4.17$ ), and the associations of both bullying others and being bullied with suicide attempts were slightly greater in boys (suicide attempts: $A O R=4.47,95 \%$ $\mathrm{CI}=3.28-6.08$ ) than in girls (suicide attempts: $\mathrm{AOR}=3.82$, $95 \% \mathrm{CI}=2.62-5.58)$.

\section{Discussion}

The present study found that a total of $18.9 \%$ of students reported having suicidal ideation, and 3.9\% reported having suicide attempts, and the results were in line with a previous study among Chinese adolescents (suicidal ideation: $16.1 \%$; suicide attempts: $3.1 \%){ }^{4}$ It has been well established that the rate of suicidal behavior was associated with the biological gender, ${ }^{7}$ and our study also demonstrated that the prevalence of suicidal ideation or suicide attempts in girls (suicidal ideation: $21.2 \%$; suicide attempts: $4.2 \%$ ) was higher than that in boys (suicidal ideation: $16.2 \%$; suicide attempts: $3.4 \%$ ).

Our univariate logistic analyses first found that adolescents who were involved in all three types of bullying (i.e., victims, perpetrators, and bully-victims) were at a higher risk of suicidal ideation and suicide attempts. After adjusting for living arrangement, age, household socioeconomic status, academic performance, classmate relations, relationships with teachers, smoking, and drinking, these associations remain statistically significant, with bully-victims having the strongest associations with suicidal ideation and suicide attempts. Similarly, Brunstein et al found that victims, bullies, and bully-victims were at a higher risk of suicidal ideation, and the most troubled adolescents were those who were bully-victims; ${ }^{25}$ Hepburn et al reported that students who reported having been involved in bullying as a perpetrator, victim, or bully-victim were more likely than those who had not been involved in bullying to report having suicidal ideation; ${ }^{26}$ Hong et al also observed independent associations of all 3 categories of bullying (perpetration, victimization, and both) with suicidal ideation among Chinese adolescents; ${ }^{12}$ Alavi et al found that a history of being bullied was a significant predictor of suicidal ideation among adolescents. ${ }^{27}$ A possible explanation for these findings might be that adolescents who bully others tend to exhibit higher levels of delinquent behaviors, ${ }^{17}$ which are reported to be related to suicidal behavior; ${ }^{9}$ adolescents involved in bullying were more likely to experience mental health problems (e.g., depression or anxiety), ${ }^{25}$ which are risk factors for suicidal behavior. ${ }^{28}$ However, there were also some studies with contrary findings. Liang et al found that victims and bullies, but not bully-victims, were more likely to consider attempting suicide; ${ }^{29}$ Klomek et al found that bullying behavior at age 8 years was not associated with suicidal ideation ten years later. ${ }^{30}$ The differences between our findings and other studies might be related to the measurement of bullying (e.g. the present study measuring the traditional bullying, but some studies measuring the cyberbullying or both traditional bullying and cyberbullying), the study design or sample size, and the nature of the samples (e.g., age or gender).

Additionally, consistent with previous evidence, ${ }^{31}$ our univariate logistic models also found that compared to boys, girls were more likely to report having suicidal ideation or suicide attempts. Furthermore, there was a significant interaction between bullying behavior and gender ( $P$ for suicidal ideation $=0.019$ and $P$ for suicide attempts $=0.015$ ), and stratification analyses across gender demonstrated that although being bullied, bullying others, and both bullying others and being bullied were positively associated with suicidal ideation and suicide attempts among both boys and girls, the associations of being bullied or bullying others with suicidal ideation and suicide attempts were slightly stronger in girls, and the associations of both being bullied and bullying others with suicidal ideation and suicide attempts were more significant in boys. These results suggested that the biological gender may modify the influences of bullying on suicidal ideation and suicide attempts among adolescents. Similarly, Klomek et al found that frequent bullying and victimization were associated with later suicide attempts among boys, and only frequent victimization was associated with suicide attempts among girls; ${ }^{18}$ Park et al reported that all 3 types of bullying behavior were predictive of suicidal ideation for males, and the only victim of bullying behavior was predictive of suicidal ideation for females. ${ }^{32}$ A possible explanation for the difference by sex is that boys were more likely to be involved as bullies, and girls were more likely to be victims. ${ }^{17}$ Another plausible explanation might be related to the different responses to bullying behavior among boys and girls, and girls have been found to more vulnerable to stress or mental health problems than boys, ${ }^{33}$ which has been found to increase the risk of suicidal behavior. ${ }^{28}$ Moreover, genetic risk of suicidal behavior (a functional variant in the serotonin transporter gene) has been observed to be particularly high for female subjects. ${ }^{34}$ Thus, being a victim or bully had a slightly strong association with suicidal ideation and suicide attempts in girls. Overall, these findings suggest some preventive recommendations against bullying and suicidal behavior 
among adolescents: (1) providing early risk assessment to adolescents who have experienced bullying behavior; (2) improving families' and schools' awareness of the adverse effects of bullying behavior; (3) establishing school-based prevention or intervention program (e.g., the Olweus Bullying Prevention Program) which have essential functions to attenuate the detrimental effects of bullying behavior. All the suggestions above are recommended to take the biological gender into consideration, and future studies are also warranted to investigate the reasons of differences by gender in the association of bullying and suicidal behavior.

Although the study findings may enhance our understanding of the correlates of suicidal ideation and suicide attempts among adolescents, this study has several limitations which should be noted. First, due to its cross-sectional study design, all reported associations should be interpreted with caution, and prospective studies are needed to estimate cause-effect associations. Second, this study used a self-report questionnaire to collect information about bullying and suicidal behavior. Although the measurements of bullying and suicidal behavior were defined with detailed questions, and this method has been widely accepted, the answers may still be subjectively biased. Third, this study only included school students, but bullying or suicidal behavior may be more common among those who were absent from school. Fourth, although in this study we mainly focused on the presence of suicidal ideation or suicide attempt among Chinese adolescents, there may be differences in characteristics between adolescents with a history of single suicidal behavior (suicidal ideation or suicide attempt) and those with multiple suicidal behavior. We may lose some information by dichotomizing the variables of suicidal ideation and suicide attempt. Fifth, due to the large sample size in this study, the clinical significance of the statistically significant interaction effects is needed to be verified by future studies.

\section{Conclusions}

In summary, bullying (bullying others, being bullied, and both bullying others and being bullied) can increase the probability of suicidal ideation and suicide attempts among Chinese adolescents, and the risk was highest in bully-victims. Moreover, compared to boys, girls were at a higher risk of suicidal ideation and suicide attempts. Further stratification analyses by gender showed that the associations of bullying others and being bullied with suicidal ideation and suicide attempts were slightly stronger in girls than those in boys, indicating that the biological gender may play a moderating role in the association of bullying with suicidal behavior. Even though the precise mechanism for the associations found in the present study is still unclear, the findings are essential for families, schools, and communities who strive to support adolescents who have been involved in bullying behavior.

\section{Disclosure}

The authors report no conflicts of interest in this work.

\section{References}

1. Nock MK, Borges G, Bromet EJ, Cha CB, Kessler RC, Lee S. Suicide and suicidal behavior. Epidemiol Rev. 2008;30:133-154. doi:10.1093/epirev/mxn002

2. Nock MK. Self-injury. Annu Rev Clin Psychol. 2010;6:339-363. doi:10.1146/annurev.clinpsy.121208.131258

3. Zhang J, Sun L, Liu Y, Zhang J. The change in suicide rates between 2002 and 2011 in China. Suicide Life Threat Behav. 2014;44 (5):560-568. doi:10.1111/sltb.2014.44.issue-5

4. Guo L, Luo M, Wang W, et al. Association between nonmedical use of opioids or sedatives and suicidal behavior among Chinese adolescents: an analysis of sex differences. Aust N Z J Psychiatry. 2019;53 (6):559-569. doi:10.1177/0004867418814944

5. Cluver L, Orkin M, Boyes ME, Sherr L. Child and adolescent suicide attempts, suicidal behavior, and adverse childhood experiences in South Africa: a prospective study. J Adolesc Health. 2015;57 (1):52-59. doi:10.1016/j.jadohealth.2015.03.001

6. World Health Organization. Preventing Suicide: A Global Imperative; 2014.

7. Cash SJ, Bridge JA. Epidemiology of youth suicide and suicidal behavior. Curr Opin Pediatr. 2009;21(5):613-619. doi:10.1097/ MOP.0b013e32833063e1

8. Adrian M, Miller AB, McCauley E, Vander SA. Suicidal ideation in early to middle adolescence: sex-specific trajectories and predictors. $J$ Child Psychol Psychiatry. 2016;57(5):645-653. doi:10.1111/jcpp.12484

9. King CA, Merchant CR. Social and interpersonal factors relating to adolescent suicidality: a review of the literature. Arch Suicide Res. 2008;12(3):181-196. doi:10.1080/13811110802101203

10. Espelage DL, Bosworth K, Simon TR. Short-term stability and prospective correlates of bullying in middle-school students: an examination of potential demographic, psychosocial, and environmental influences. Violence Vict. 2001;16(4):411-426. doi:10.1891/0886-6708.16.4.411

11. Hertz MF, Donato I, Wright J. Bullying and suicide: a public health approach. J Adolesc Health. 2013;53(1):S1-S3. doi:10.1016/j. jadohealth.2013.05.002

12. Hong L, Guo L, Wu H, et al. Bullying, depression, and suicidal ideation among adolescents in the Fujian Province of China: a cross-sectional study. Medicine (Baltimore). 2016;95(5):e2530. doi:10.1097/MD.0000000000002530

13. Herba CM, Ferdinand RF, Stijnen T, et al. Victimization and suicide ideation in the TRAILS study: specific vulnerabilities of victims. J Child Psychol Psychiatry. 2008;49(8):867-876.

14. Espelage DL, Holt MK. Suicidal ideation and school bullying experiences after controlling for depression and delinquency. $J$ Adolesc Health. 2013;53(1 Suppl):S27-S31. doi:10.1016/j.jadohealth.2012.09.017

15. $\mathrm{Wu} \mathrm{J}, \mathrm{He} \mathrm{Y,} \mathrm{Lu} \mathrm{C}$, et al. Bullying behaviors among Chinese school-aged youth: a prevalence and correlates study in Guangdong Province. Psychiatry Res. 2015;225(3):716-722. doi:10.1016/j. psychres.2014.11.004

16. Kaltiala-Heino R, Rimpela M, Marttunen M, Rimpela A, Rantanen P. Bullying, depression, and suicidal ideation in Finnish adolescents: school survey. BMJ. 1999;319(7206):348-351. doi:10.1136/bmj.319.7206.348 
17. Vieno A, Gini G, Santinello M. Different forms of bullying and their association to smoking and drinking behavior in Italian adolescents. $J$ Sch Health. 2011;81(7):393-399. doi:10.1111/j.1746-1561.2011.00607.x

18. Klomek AB, Sourander A, Niemela S, et al. Childhood bullying behaviors as a risk for suicide attempts and completed suicides: a population-based birth cohort study. J Am Acad Child Adolesc Psychiatry. 2009;48 (3):254-261. doi:10.1097/CHI.0b013e318196b91f

19. Laukkanen E, Honkalampi K, Hintikka J, Hintikka U, Lehtonen J. Suicidal ideation among help-seeking adolescents: association with a negative self-image. Arch Suicide Res. 2005;9(1):45-55. doi:10.1080/13811110590512930

20. Kim YS, Leventhal BL, Koh YJ, Boyce WT. Bullying increased suicide risk: prospective study of Korean adolescents. Arch Suicide Res. 2009;13(1):15-30. doi:10.1080/13811110802572098

21. Guo L, Xu Y, Deng J, et al. Association between sleep duration, suicidal ideation, and suicidal attempts among Chinese adolescents: the moderating role of depressive symptoms. J Affect Disord. 2017;208:355-362. doi:10.1016/j.jad.2016.10.004

22. Zwald ML, Annor FB, Wilkinson A, et al. Suicidal ideation and attempts among students in grades 8, 10, and 12 - Utah, 2015. MMWR Morb Mortal Wkly Rep. 2018;67(15):451-454. doi:10.15585/mmwr.mm6715a4

23. Wang $\mathrm{H}$, Zhou X, Lu C, et al. Adolescent bullying involvement and psychosocial aspects of family and school life: a cross-sectional study from Guangdong Province in China. PLOS ONE. 2012;7(7):e38619. doi:10.1371/journal.pone.0038619

24. Guo L, Luo M, Wang W, Huang G, Zhang WH, Lu C. Association between weekday sleep duration and nonmedical use of prescription drug among adolescents: the role of academic performance. Eur Child Adolesc Psychiatry. 2019;28(9):1265-1275. doi:10.1007/s00787-01901294-9

25. Brunstein KA, Marrocco F, Kleinman M, Schonfeld IS, Gould MS. Bullying, depression, and suicidality in adolescents. J Am Acad Child Adolesc Psychiatry. 2007;46(1):40-49. doi:10.1097/01.chi. 0000242237.84925 .18
26. Hepburn L, Azrael D, Molnar B, Miller M. Bullying and suicidal behaviors among urban high school youth. J Adolesc Health. 2012;51 (1):93-95. doi:10.1016/j.jadohealth.2011.12.014

27. Alavi N, Reshetukha T, Prost E, et al. Relationship between bullying and suicidal behaviour in youth presenting to the emergency department. J Can Acad Child Adolesc Psychiatry. 2017;26(2):70-77.

28. Joffe BI, Van Lieshout RJ, Duncan L, Boyle MH. Suicidal ideation and behavior in adolescents aged 12-16 years: a 17-year follow-up. Suicide Life Threat Behav. 2014;44(5):497-509. doi:10.1111/sltb.2014.44.issue-5

29. Liang H, Flisher AJ, Lombard CJ. Bullying, violence, and risk behavior in South African school students. Child Abuse Negl. 2007;31(2):161-171. doi:10.1016/j.chiabu.2006.08.007

30. Klomek AB, Sourander A, Kumpulainen K, et al. Childhood bullying as a risk for later depression and suicidal ideation among Finnish males. $J$ Affect Disord. 2008;109(1-2):47-55. doi:10.1016/j.jad.2007.12.226

31. Stoep AV, McCauley E, Flynn C, Stone A. Thoughts of death and suicide in early adolescence. Suicide Life Threat Behav. 2009;39 (6):599-613. doi:10.1521/suli.2009.39.6.599

32. Park HS, Schepp KG, Jang EH, Koo HY. Predictors of suicidal ideation among high school students by gender in South Korea. J Sch Health. 2006;76(5):181-188. doi:10.1111/j.1746-1561.2006.00092.x

33. Bond L, Carlin JB, Thomas L, Rubin K, Patton G. Does bullying cause emotional problems? A prospective study of young teenagers. BMJ. 2001;323(7311):480-484. doi:10.1136/bmj.323.7311.480

34. Baca-Garcia E, Vaquero C, Diaz-Sastre C, Saiz-Ruiz J, FernandezPiqueras J, de Leon J. A gender-specific association between the serotonin transporter gene and suicide attempts. Neuropsychopharmacol. 2002;26 (5):692-695. doi:10.1016/S0893-133X(01)00394-3
Psychology Research and Behavior Management

\section{Publish your work in this journal}

Psychology Research and Behavior Management is an international, peer-reviewed, open access journal focusing on the science of psychology and its application in behavior management to develop improved outcomes in the clinical, educational, sports and business arenas. Specific topics covered in the journal include: Neuroscience, memory and decision making; Behavior modification and management; Clinical applications; Business and sports performance management; Social and developmental studies; Animal studies. The manuscript management system is completely online and includes a very quick and fair peer-review system, which is all easy to use. Visit http://www dovepress.com/testimonials.php to read real quotes from published authors. 